\title{
Rechte Orientierungen als Herausforderung professionellen Handelns
}

\section{Situationsbeschreibungen aus der Praxis der Kinder- und Jugendhilfe}

\author{
PROF. DR. MICHAEL GÖRTLER \\ lehrt Theorien und Geschichte \\ der Sozialen Arbeit an der \\ Fakultät Angewandte Sozial- und \\ Gesundheitswissenschaften der \\ OTH Regensburg.
}

michael.goertler@oth-regensburg.de

\author{
Mitarbeitende in sämtlichen Feldern der Sozialen \\ Arbeit sehen sich zunehmend mit rechtspopulistischen \\ und auch rechtsextremen Einstellungen konfrontiert, \\ mit denen angemessen umzugehen eine enorme \\ Herausforderung ist. Das gilt in besonderem \\ Maße auch für die Kinder- und Jugendhilfe.
}

Aktuelle Bevölkerungsumfragen zeigen, dass rechte Orientierungen - als Sammelbegriff für Einstellungen, die als rechtsextremh, menschenfeindlich, rechtspopulistisch, antidemokratisch usw. eingeordnet werden - in allen Teilen der Gesellschaft beheimatet sind (vgl. etwa Zick et al. 2019). So belegt die »Mitte-Studie « der Friedrich-EbertStiftung, dass sich rechtspopulistische Einstellungen in der Mitte der Gesellschaft verfestigt haben, wobei die Abwertung von Geflüchteten weit verbreitet ist (FES o.J.). Als Randphänomen betrachtet, wurde die Verbreitung von Rechtsextremismus, Gruppenbezogener Menschenfeindlichkeit, Rechtspopulismus usw. bis vor wenigen Jahren in Öffentlichkeit und Politik systematisch unterschätzt - ein einschlägiges Beispiel dafür sind die sog. NSU-Morde, deren Aufarbeitung und die damit verbundenen Debatten. Auch im Fachdiskurs der Sozialen Arbeit ist dieser Problemkomplex erst in den letzten Jahren aus einer Nische heraus in den Fokus der Aufmerksamkeit gerückt (vgl. etwa Kleffner 2015, Buttner 2020). Diesem Befund steht der dringende Handlungs-, aber auch Forschungsbedarf gegenüber, nicht zuletzt, weil die gesellschaftliche Lage angespannt ist (z.B. Gründung rechtspopulistischer Bewegungen, rechtsextrem motivierte Anschläge auf Einrichtungen und Attentate auf Personen, antidemokratische und menschenfeindliche Stimmungen in der Bevölkerung im Zuge der COVID-19-Pandemie).

Aus den genannten Gründen muss davon ausgegangen werden, dass rechte Orientierungen auch im Wirkungskreis der Sozialen Arbeit eine Rolle spielen. Dabei ist die Dunkelziffer als hoch einzuschätzen, denn es sind zwar einzelne Berichte über Unterwanderungsversuche in sozialen Einrichtungen seitens der rechten Szene sowie einzelne Studien zur rechten Einstellungen von Fachkräften, Adressat*innen, Studierenden usw. vorhanden (vgl. etwa Sozialmagazin 2017; Forum Jugendarbeit 2020), eine systematische Erfassung dieses Problemkomplexes steht aber noch aus. Es liegt auf der Hand, dass es sich dabei um eine Herausforderung professionellen Handelns auf mehreren Ebenen handelt, also nicht nur von außen, sondern auch von innen. Die Professionalität der Fachkräfte wird auf die Probe gestellt, denn Denk- und Handlungsweisen, die auf eine rechte Orientierung schließen lassen, sind mit der Berufsethik, insbesondere der Bekenntnis zu den Grund- und Menschenrechten sowie zur freiheitlichdemokratischen Grundordnung und den damit verbundenen Werten und Prinzipien, unvereinbar (vgl. DBSH 2014). Vor diesem normativen Hintergrund muss 
das professionelle Handeln der Fachkräfte in der Praxis darauf ausgerichtet sein, der gruppenbezogenen Abwertung entgegenzuwirken. Mit Blick auf den Entscheidungsdruck sowie die Unwägbarkeiten, die typisch für die sozialarbeiterische Handlungspraxis sind (vgl. Dewe/Otto 2012) lässt dieser Anspruch die Reflexion und Beurteilung des (eigenen) Handelns, aber auch des Handelns der Interaktionspartner*innen (z.B. Adressat*innen, Eltern, Kolleg*innen) sowie der Kultur in der (eigenen) sozialen Einrichtung als Basis für eine professionelle Intervention zur Herausforderung werden. Dabei müssen die Fachkräfte auf verschiedene Ebenen Einfluss (z.B. Sozialraum, Gesellschaft) ausüben, wenn sie ihre Umwelt mitgestalten wollen.

Im Folgenden soll zunächst das Begriffsfeld umrissen und dann anhand von vier ausgewählten Situationsbeschreibungen veranschaulicht werden, welche Herausforderungen professionellen Handelns in diesem Kontext in der Praxis auftreten können. Die Basis dafür bildet eine klein angelegte Befragung von Fachkräften, die in der Kinder- und Jugendhilfe tätig sind, zu Erfahrungen mit Personen mit rechten Orientierungen bzw. im Kontext von Rechtsextremismus und Rechtspopulismus am Arbeitsplatz.

\section{Zur (schwierigen) Einordnung der Begriffe zwischen Theorie und Praxis}

Das Begriffsfeld um rechte Orientierungen bzw. Rechtsextremismus, Rechtspopulismus und den damit verbundenen Einstellungen ist aufgrund konkurrierender Konzepte und Methoden zur Messung nicht trennscharf, so dass bereits die Einordnung von Denk- und Handlungsweisen zur Herausforderung wird. Wenngleich die Abgrenzung der Begriffe nicht Gegenstand dieses Beitrags ist, erscheint es sinnvoll, in der gebotenen Kürze zu umreißen, was damit gemeint sein kann.

In Öffentlichkeit und Politik wird rege über rechte Orientierungen als Gefahr für die Demokratie diskutiert, wobei die Konturen im Begriffsfeld verschwimmen. So ist beispielsweise von rechten Einstellungen, von rechtem Gedankengut, einer rechten Gesinnung, einem rechten Weltbild usw., von Rechtsextremismus, Rechtsradikalismus, (Neo-)Nazismus, Ausländerfeindlichkeit usw. die Rede (vgl. Stöss 2010). Auch für die Wissenschaft stellen Stöss (vgl. 2010) und Spier (vgl. 2014) fest, dass unterschiedliche Definitionen von Rechtsextremismus und Rechtspopulismus sowie den damit verbundenen Einstellungen kursieren. Kiess et al. 2015 resümieren, dass »die Ideologie der Ungleichwertigkeit« eine Gemeinsamkeit im Begriffsfeld darstellt.

Eben genannte Ideologie der Ungleichwertigkeit bezieht sich nicht nur auf einzelne Personen, sondern auf Personengruppen und gehört zum »Syndrom der Gruppenbezogenen Menschenfeindlichkeit « das u. a. die folgenden Elemente umfasst: "Fremdenfeindlichkeit und die Befürwortung ganz allgemein von Etabliertenvorrechten für Alteingesessene im Vergleich zu Neuankömmlingen, ethnischer Rassismus, Antisemitismus, Sexismus, die Abwertung von Muslimen, von Sinti und Roma und asylsuchenden Menschen sowie von homosexuellen, behinderten, obdachlosen und langzeitarbeitslosen Menschen« (Küpper/Zick 2015). Der Rechtspopulismus wird als "Politikstil « und »Ideologie « definiert (Spier 2014), der sich einige Elemente des Rechtsextremismus und der Gruppenbezogenen Menschenfeindlichkeit zu eigen macht (z. B. Abwertung von Geflüchteten), andere dagegen nicht (z. B. Ablehnung der Demokratie).

Resümierend ist das Begriffsfeld in der Theorie nicht trennscharf und die Grenzen zwischen den Konzepten und Methoden zur Messung von Einstellungen sind fließend. Wie schwer die Einordnung fällt, zeigen nicht zuletzt die öffentlich geführten Debatten um den sog. Rechtsruck in Politik und Gesellschaft, die (Nicht-) Abgrenzung rechtspopulistischer zu (neu-) rechten Bewegungen oder die Einstufung von Parteien und Politikern als rechtspopulistisch und/oder rechtsextrem. Dieser Umstand lässt die Einordnung der Begriffe in der Praxis zur Herausforderung werden und es darf nicht verwundern, dass sich die Unklarheiten auch in den Situationsbeschreibungen der Fachkräfte widerspiegeln. Daher wurde in dieser klein angelegten Befragung keine Differenzierung vorgenommen, um bestimmte Einstellungen zu messen, sondern ganz allgemein nach Erfahrungen mit Personen mit rechten Orientierungen bzw. im Kontext von Rechtsextremismus und Rechtspopulismus am Arbeitsplatz gefragt. Hier könnten Fortbildungen helfen, um Fachkräfte bei der Einordnung und beim Umgang mit diesem Problemkomplex zu unterstützen.

\section{Vier Situationsbeschreibungen aus der Praxis}

In diesem Abschnitt werden Herausforderungen aus der Praxis der Kinder- und Jugendhilfe anhand von exemplarischen Situationsbeschreibungen diskutiert. Die Basis dafür bildet eine inhaltsanalytisch ausgewertete Befragung von Fachkräften, die in diesem Handlungsfeld tätig sind, zu Erfahrungen mit Personen mit rechten Orientierungen bzw. im Kontext von Rechtsextremismus und Rechtspopulismus am Arbeitsplatz. Die Ergebnisse der Befragung sind nicht generalisierbar und stellen nur einen Ausschnitt der Praxiswirklichkeit dar. Daher wird an dieser Stelle ausdrücklich darauf hingewiesen, dass einige der Befragten davon berichten, dass es bis dato wenige bis keine derartigen Vorfälle in ihrer Einrichtung gegeben habe.

Die folgenden vier Situationsbeschreibungen sind kategorisiert, sowohl im paraphrasierten als auch im originalen Wortlaut wiedergegeben, um den ausgewerteten Inhalt kurz und prägnant darzustellen. In allen Situationsbeschreibungen spiegelt sich die gruppenbezogene Abwertung von Personen aufgrund von äußerlichen Merkmalen (z.B. Migrationshintergrund, der tatsächlich oder vermeintlich besteht und dabei am Aussehen, am Namen oder der Aussprache festgemacht wird) wider, die unter dem gemeinsamen Dach einer Ideologie der Ungleichwertigkeit für rechte Orientierungen bzw. Rechtsextremismus, Rechtspopulismus und den damit verbundenen Einstellungen charakteristisch ist. Dabei verlangt die Bewältigung dieser Herausforderung von den Fachkräften die Fähigkeit und Bereitschaft zu Reflexion und Beurteilung der eigenen Denkund Handlungsweise, aber auch derer der Interaktionspartner*innen sowie der Kultur in der sozialen Einrichtung als Basis für eine professionelle Intervention.

\section{Erscheinungsbild und Herkunft als (vermeintlicher) Grund für eine Behinderung}

Eine mögliche Herausforderung besteht darin, dass innerhalb der Bezugsgruppe in der Einrichtung junge Menschen von anderen jungen Menschen abgewertet 
werden. In diesem Kontext gibt eine befragte Fachkraft, die mit Kindern mit und ohne Behinderung arbeitet, an, dass sie einen Jungen betreue, der aufgrund seines Erscheinungsbilds von den anderen Kindern in der Bezugsgruppe als nicht-deutsch eingeordnet und dessen Behinderung mit der nicht-deutschen Herkunft begründet werde. Sie führt dazu aus: »Vereinzelte Kinder treffen immer wieder Aussagen, dass er [der Junge mit Behinderung, M.G.] komisch aussieht, nicht deutsch und deswegen behindert ist." In diesem Fall kommt erschwerend hinzu, dass sich diese Situation im Kontext der Sozialen Arbeit nicht nur aus der Perspektive gruppenbezogener Abwertung, sondern auch aus der der Intersektionalität (vgl. Bronner/ Paulus 2017) - im Sinne einer doppelten Diskriminierungskategorie (nicht-deutsche Herkunft und Behinderung und ggf. damit verbundene doppelte Diskriminierungserfahrung) - betrachten lässt. Solche oder ähnliche Situationen erfordern von den Fachkräften einen sensiblen Umgang mit den Betroffen, um mit Blick auf eine nachhaltige Veränderung der Gruppendynamik die Sach- und Beziehungslage klären, Grenzen setzen, Maßnahmen treffen zu können usw.

\section{Leichtsinn, Ungewissheit oder echte Überzeugung}

Eine andere mögliche Herausforderung ergibt sich daraus, dass ein Jugendlicher Denk- und Handlungsweisen, die auf eine rechte Orientierung schließen lassen, an den Tag legt, wobei seitens der Fachkraft zu klären ist, welche Motive dabei zugrunde liegen. Hier berichtet eine Fachkraft von zwei Vorfällen: »Ein zehnjähriger Bewohner [in der Einrichtung der befragten Person, M.G.] ist dunkelhäutig. Hier kommt es oft zu Anspielungen auf seine Hautfarbe von den anderen Mitbewohnern. Dies werte ich aber nicht als Rechtsextremismus, sondern als jugendliche Unwissenheit ... Einen 16-jährigen Jugendlichen habe ich mal zu einer Gerichtsverhandlung begleitet. Er war wegen der Verwendung verfassungswidriger Kennzeichen angeklagt. Auch hier würde ich nicht von Rechtsextremismus sprechen, da dies auch eher aus jugendlichem Leichtsinn geschehen ist und der Jugendliche keineswegs menschenfeindlich ist.«Die Fachkraft ist sich in beiden Fällen zwar sicher, aufgrund ihrer Erfahrung mit ihrer Einschätzung der Motive der Jugendlichen richtig zu liegen, versteht sich selbst aber nicht als Experte für den Problemkomplex. Vor dem Hintergrund der Professionalität sollte die Einordnung der Denk- und Handlungsweisen der Jugendlichen nicht nur auf der eigenen Menschenkenntnis, sondern auch auf fachlichen Konzepten basieren, um beispielsweise durch das Nähe-DistanzVerhältnis bedingte - Fehleinschätzungen zu vermeiden.

\section{Ressentiments in der Elternschaft}

Wieder eine andere mögliche Herausforderung schlägt sich in der Interaktion mit den Eltern nieder. Ein erstes Beispiel bezieht sich auf die ehrenamtliche Tätigkeit einiger Fachkräfte einer Einrichtung im Rahmen der Flüchtlingshilfe, insbesondere einer Spendenaktion für geflüchtete junge Menschen sowie deren Unterbringung bei den Fachkräften zuhause in Not- und daher Ausnahmefällen. Dieses vor dem normativen Hintergrund der Berufsethik der Sozialen Arbeit ( $\rightarrow$ Menschenrechtsprofession) geleistete bürgerschaftliche Engagement wurde laut der Fachkraft von einigen Eltern, deren Kinder in der Einrichtung betreut werden, in Gestalt der gruppenbezogenen Abwertung kritisiert. Die Fachkraft berichtet von Fragen und Kommentaren, wie »warum wir denn hier ssolches Gesocks` aufnehmen ... wahrscheinlich auch noch kostenlos ... da wird so viel Geld reingesteckt, aber für unsere Kinder ist kein Geld da in Deutschland." In diesem Kontext kann an den Ursachen von Rechtsextremismus und Rechtspopulismus bzw. rechten Einstellungen, aber auch am Konzept des sog. Nützlichkeitsrassismus angesetzt werden, weil die subjektiv wahrgenommene bzw. in Relation gesetzte eigene ökonomische Lage betont und damit eine Schlechterstellung angedeutet wird (vgl. Kiess et al. 2015). Ein zweites, ähnlich gelagertes Beispiel bezieht sich auf die - seitens des Trägers ausdrücklich erwünschte - interkulturelle Öffnung der Einrichtung durch Bräuche, Feste, Feiertage usw., die in anderen Religionen üblich sind. Hier berichtet die Fachkraft von Fragen und Kommentaren, wie »wo kommen wir denn da hin, wollt ihr jetzt auch nur noch Lichterfest feiern und Nikolaus ausfallen lassen? Wir sind eine katholische Einrichtung in Deutschland! «Solche und ähnliche Situationen verlangen von der einzelnen Fachkraft und der ganzen Einrichtung, eine klare Haltung nach außen zu demonstrieren, die auch vom Träger kommuniziert werden muss, um in der Interaktion mit den Eltern geschlossen auftreten zu können.

\section{Ressentiments (auch) im Kollegium}

Eine letzte mögliche Herausforderung resultiert aus einer Abwertung, die von den Fachkräften selbst gegenüber anderen Personen (z. B. anderen Fachkräften, Eltern) ausgeht. Hier berichtet eine Fachkraft von einem Vorfall im Kontext einer Stellenbesetzung: »Bei der Besprechung einer Bewerbung eines Mannes mit schwarzer Hautfarbe kam die Bemerkung >Im Dunkeln siehst du da nur noch die Zähne`." Diese Aussage wurde nach Angabe der Fachkraft von den anwesenden Kolleg*innen zum Teil belächelt, zum Teil ignoriert - eine Reaktion im Sinne der Demonstration einer klaren Haltung gegen diese gruppenbezogene Abwertung blieb aber aus. Mit Blick auf die Professionalität der Fachkräfte werden hier zwei Dinge sichtbar: Zum einen, dass eine Fachkraft eine solche Aussage (überhaupt) nicht treffen sollte, zum anderen, dass die anwesenden Fachkräfte ein solches Verhalten nicht akzeptieren sollten. Eine andere, ähnlich gelagerte Situation betrifft das Verhalten der Fachkräfte gegenüber Eltern. Ein Beispiel ist der »unprofessionelle Umgang mit Zweisprachigkeit", den die davon berichtende Fachkraft wie folgt beschreibt: "Dieser zeigt sich bei Abholsituationen durch Kommentare von Mitarbeitern gegenüber Eltern, wie etwa \Sprechen Sie doch nicht mit ihrem Kind türkisch/russisch/ polnisch, sondern deutsch!« Eine andere Fachkraft gibt an, dass sich eine Kollegin nach einer Kontroverse mit einer Mutter mit Migrationshintergrund wie folgt äußerte: "Da braucht man sich aber nicht mehr wundern, dass der Ausländerhass immer größer wird! « Diese Situationen zeigen, dass rechte Orientierungen auch im Kollegium vorkommen können und - möglicherweise - von den Fachkräften selbst oder von den Kolleg*innen als gemäßigt und nicht extrem und damit als akzeptabel wahrgenommen werden, obwohl sie fraglos eine gruppenbezogene Abwertung darstellen. 


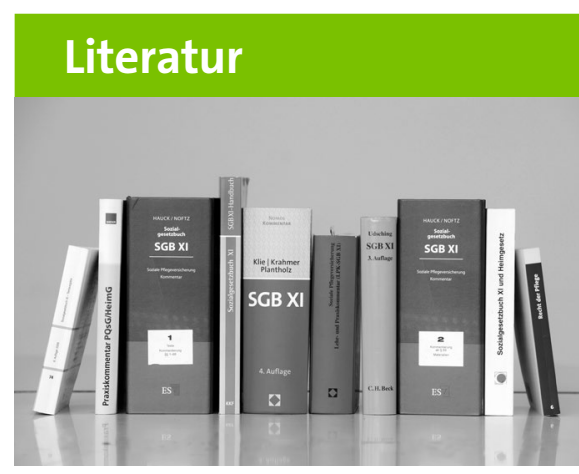

Bronner, K. / Paulus, S. (2017):

Intersektionalität. Geschichte, Theorie und Praxis. Opladen, Berlin, Toronto.

Buttner, P. (2020): Soziale Arbeit und Rechtsextremismus. Archiv für Wissenschaft und Praxis der sozialen Arbeit. Nr. 2/2020. DBSH (2014): Berufsethik des DBSH. Ethik und Werte. In: FORUM sozial. Die Berufliche Soziale Arbeit. 4/2012.

Dewe, B. / Otto, H.-U. (2018): Professionalität. In: Otto, H.-U./Thiersch, H. (Hg.): Handbuch
Soziale Arbeit: Grundlagen der Sozialarbeit und Sozialpädagogik. 4., völlig neu bearbeitete Auflage. München und Basel. S. 1203-1213.

FES (Friedrich-Ebert-Stiftung) (o.J.):

Mitte-Studie. Online verfügbar unter:

https://www.fes.de/forum-berlin/gegen-

rechtsextremismus/mitte-studie/ (Zugriff am 27.11.2020)

Forum für Kinder- und Jugendarbeit (2020):

Rechts(d)ruck in Sozialer Arbeit. 36. Jg.

2. Quartal.

Kiess, J. /Decker, O. / Brähler, E. (2015): Was ist rechtsextreme Einstellung, und woraus besteht sie? Veröffentlicht am 13.1.2015. URL: https://www.bpb.de/politik/extremismus/ rechtsextremismus/198945/was-istrechtsextreme-einstellung-und-worausbesteht-sie (Zugriff am 27.11.2020)

Kleffner, H. (2015): Die Leerstelle in der Fachdiskussion füllen. Sozialarbeit und der NSU-Komplex. URL: https://www.bpb. de/apuz/212367/sozialarbeit-und-der-nsukomplex?p=all (Zugriff am 27.11.2020)
Küpper, R. / Zick, A. (2015): Gruppenbezogene Menschenfeindlichkeit. URL: https:// www.bpb.de/politik/extremismus/ rechtsextremismus/214192/ gruppenbezogene-menschenfeindlichkeit (Zugriff am 27.11.2020).

Sozialmagazin (2017): Rechtspopulismus und Soziale Arbeit. H. 11-12.

Spier, T. (2014): Was versteht man unter „Populismus“. URL https:// www.bpb.de/politik/extremismus/ rechtsextremismus/192118/was-verstehtman-unter-populismus (Zugriff am 27.11.2020)

Stöss, R. (2010): Rechtsextremismus im Wandel. URL: library.fes.de/pdf-files/ do/08223.pdf (Zugriff am 27.11.2020) Zick, A. / Küpper, B. / Berghan, W. (2019): Verlorene Mitte - Feindselige Zustände. Rechtsextreme Einstellungen in Deutschland 2018/19. Hg. für die Friedrich-Ebert-Stiftung v. Franziska Schröte. Bonn.

\section{Extremismus und Soziale Arbeit}

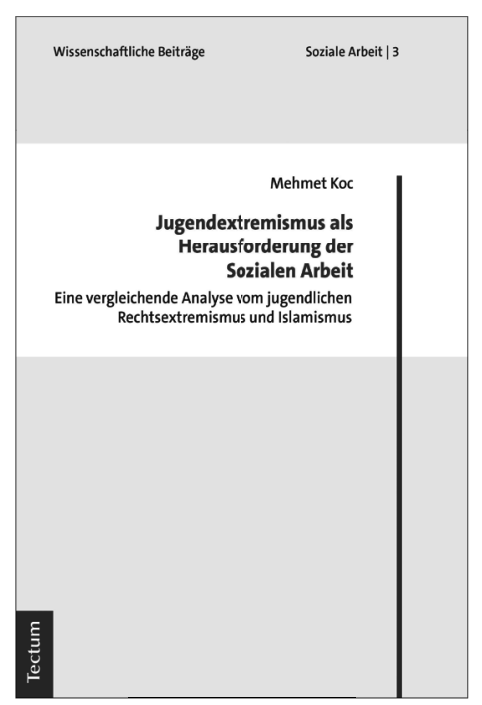

Jugendextremismus als

Herausforderung der Sozialen Arbeit

Eine vergleichende Analyse vom jugendlichen Rechtsextremismus und Islamismus

(Wissenschaftliche Beiträge aus dem Tectum Verlag: Soziale Arbeit, Bd. 3)

2019, 144 S., brosch.,

Print 20,00€ •E-Book 15,99 €

ISBN 978-3-8288-4361-5

ePDF 978-3-8288-7316-2

Extremismus gefährdet Jugendliche in vielerlei Hinsicht und gewinnt zunehmend wieder an Bedeutung für die Soziale Arbeit. Durch die Entwicklung von extremistischen Einstellungsmustern gefährden Jugendliche dabei sich selbst und andere Personen zugleich. Es lässt sich beobachten, dass demokratische Grundwerte bei Jugendlichen sukzessive an Bedeutung verlieren. Aus dem pädagogischen Auftrag zur Erziehung von Kindern und Jugendlichen zu „eigenverantwortlichen und gemeinschaftsfähigen Persönlichkeiten" (\$1 SGB VIII) ergibt sich die Aufgabe der Sozialen Arbeit, sich mit dem Jugendextremismus intensiv zu befassen. 